\title{
On Harmonic Functions Defined by Differential Operator with Respect to $k$-Symmetric Points
}

\author{
Afaf A. Ali Abubaker and Maslina Darus \\ School of Mathematical Sciences, Faculty of Science and Technology, Universiti Kebangsaan Malaysia, 43600 Bangi, \\ Selangor D. Ehsan, Malaysia
}

Correspondence should be addressed to Maslina Darus; maslina@ukm.edu.my

Received 29 April 2014; Revised 4 July 2014; Accepted 7 July 2014; Published 23 July 2014

Academic Editor: Heinrich Begehr

Copyright ( 2014 A. A. A. Abubaker and M. Darus. This is an open access article distributed under the Creative Commons Attribution License, which permits unrestricted use, distribution, and reproduction in any medium, provided the original work is properly cited.

We introduce new classes $M H_{k}^{\sigma, s}(\lambda, \delta, \alpha)$ and $\bar{M} H_{k}^{\sigma, s}(\lambda, \delta, \alpha)$ of harmonic univalent functions with respect to $k$-symmetric points defined by differential operator. We determine a sufficient coefficient condition, representation theorem, and distortion theorem.

\section{Introduction}

A continuous function $f=u+i v$ is a complex valued harmonic function in a complex domain $C$ if both $u$ and $v$ are real harmonic in $C$. In any simply connected domain $D \subset C$ we can write $f(z)=h+\bar{g}$, where $h$ and $g$ are analytic in $D$. We call $h$ the analytic part and $g$ the coanalytic part of $f$. A necessary and sufficient condition for $f$ to be locally univalent and sense preserving in $D$ is that $\left|h^{\prime}(z)\right|>\left|g^{\prime}(z)\right|$ in $D$. See Clunie and Shell-Small (see [1]).

Thus for $f=h+\bar{g} \in S^{*} H$, we may write

$$
h(z)=z+\sum_{n=2}^{\infty} a_{n} z^{n}, \quad g(z)=\sum_{n=1}^{\infty} b_{n} z^{n}, \quad\left|b_{1}\right|<1 .
$$

Note that $S^{*} H$ reduces to $S^{*}$, the class of normalized analytic univalent functions if the coanalytic part of $f=h+\bar{g}$ is identically zero. Also, denote by $S H$ the subclasses of $S^{*} H$ consisting of functions $f$ that map $U$ onto starlike domain.

A function $f$ is said to be starlike of order $\alpha$ in $U$ denoted by $S H(\alpha)$ (see [2]) if

$$
\begin{aligned}
\frac{\partial}{\partial \theta}\left(\arg f\left(r e^{i \theta}\right)\right) & =\operatorname{Im}\left\{\frac{(\partial / \partial \theta) f\left(r e^{i \theta}\right)}{f\left(r e^{i \theta}\right)}\right\} \\
& =\Re\left\{\frac{z h^{\prime}(z)-\overline{z g^{\prime}(z)}}{h(z)+\overline{g(z)}}\right\} \geq \alpha, \\
|z|=r & <1 .
\end{aligned}
$$

A function $f$ of normalized univalent analytic functions is said to be starlike with respect to symmetrical points in $U$ if it satisfies

$$
\Re\left\{\frac{z f^{\prime}(z)}{f(z)-f(-z)}\right\}>0, \quad z \in U
$$

this class was introduced and studied by Sakaguchi in 1959 [3]. Some related classes are studied by Shanmugam et al. [4].

In 1979, Chand and Singh [5] defined the class of starlike functions with respect to $k$-symmetric points of order $\alpha(0 \leq$ $\alpha<1)$. Related classes are also studied by das and Singh [6]. Ahuja and Jahangiri [7] discussed the class $S H(\alpha)$ which denotes the class of complex-valued, sense-preserving, harmonic univalent functions $f$ of the form (1) and satisfying the condition

$$
\operatorname{Im}\left\{\frac{2(\partial / \partial \theta) f\left(r e^{i \theta}\right)}{f\left(r e^{i \theta}\right)-f\left(-r e^{i \theta}\right)}\right\} \geq \alpha .
$$

In [8], the authors introduced and studied the class $S H_{k}(\alpha)$ which denotes the class of complex-valued, sense-preserving, harmonic univalent functions $f$ of the form (1) and

$$
\begin{aligned}
& h_{k}(z)=z+\sum_{n=2}^{\infty} \phi_{n} a_{n} z^{n}, \\
& g_{k}(z)=\sum_{n=1}^{\infty} \phi_{n} b_{n} z^{n}, \quad\left|b_{1}\right|<1,
\end{aligned}
$$


where

$$
\phi_{n}=\frac{1}{k} \sum_{\nu=0}^{k-1} \varepsilon^{(n-1) \nu}, \quad\left(k \geq 1, \varepsilon^{k}=1\right) .
$$

From the definition of $\phi_{n}$ we know

$$
\phi_{n}=\left\{\begin{array}{ll}
1, & n=\imath k+1, \\
0, & n \neq \iota k+1,
\end{array} \quad(n \geq 2, \iota, k \geq 1) .\right.
$$

The differential operator $D_{\lambda, \delta}^{\sigma, s}$ was introduced by Ali Abubaker and Darus [9]. We define the differential operator of the harmonic function $f=h+\bar{g}$ given by (5) as

$$
D_{\lambda, \delta}^{\sigma, s} f(z)=D_{\lambda, \delta}^{\sigma, s} h(z)+(-1)^{s} \overline{D_{\lambda, \delta}^{\sigma, s} g(z)},
$$

where

$$
\begin{aligned}
& D_{\lambda, \delta}^{\sigma, s} h(z)=z+\sum_{n=2}^{\infty} \psi_{n}(\lambda, \delta, \sigma, s) a_{n} z^{n}, \\
& D_{\lambda, \delta}^{\sigma, s} g(z)=\sum_{n=1}^{\infty} \psi_{n}(\lambda, \delta, \sigma, s) b_{n} z^{n}
\end{aligned}
$$

and also $\psi_{n}(\lambda, \delta, \sigma, s)=n^{s}(C(\delta, n)[1+\lambda(n-1)])^{\sigma}, \lambda \geq 0$, $C(\delta, n)=(\delta+1)_{n-1} /(n-1) !$, for $\delta, \sigma, s \in N_{0}=\{0,1,2, \ldots\}$, and $(x)_{n}$ is the Pochhammer symbol defined by

$$
\begin{aligned}
(x)_{n} & =\frac{\Gamma(x+n)}{\Gamma(x)} \\
& = \begin{cases}1, & n=0 \\
x(x+1) \cdots(x+n-1), & n=\{1,2,3, \ldots\} .\end{cases}
\end{aligned}
$$

We note that when $s=0, \sigma=1$, and $\lambda=0$ we obtain the Ruscheweyh derivative for harmonic functions (see [7]), when $\sigma=0$ we obtain the Salagean operator for harmonic functions (see [10]), and when $\sigma=1, s=0$ we obtain the operator for harmonic functions given by Al-Shaqsi and Darus [11].

Let $M H_{k}^{\sigma, s}(\lambda, \delta, \alpha)$ denote the class of complex-valued, sense-preserving, harmonic univalent functions $f$ of the form (5) which satisfy the condition

$$
\begin{aligned}
& \operatorname{Im}\left\{\frac{(\partial / \partial \theta) D_{\lambda, \delta}^{\sigma, s} f\left(r e^{i \theta}\right)}{D_{\lambda, \delta}^{\sigma, s} f_{k}\left(r e^{i \theta}\right)}\right\} \\
& =\Re\left\{\frac{z\left(D_{\lambda, \delta}^{\sigma, s} h(z)\right)^{\prime}-(-1)^{s} z\left(D_{\lambda, \delta}^{\sigma, s} g(z)\right)^{\prime}}{D_{\lambda, \delta}^{\sigma, s} h_{k}(z)+(-1)^{s} \overline{D_{\lambda, \delta}^{\sigma, s} g_{k}(z)}}\right\} \geq \alpha,
\end{aligned}
$$

where $z=r e^{i \theta}, 0 \leq r<1,0 \leq \theta<\pi, 0 \leq \alpha<1$ and the functions $D_{\lambda, \delta}^{\sigma, s} h_{k}$ and $D_{\lambda, \delta}^{\sigma, s} g_{k}$ are of the form

$$
\begin{aligned}
& D_{\lambda, \delta}^{\sigma, s} h_{k}(z)=z+\sum_{n=2}^{\infty} \psi_{n}(\lambda, \delta, \sigma, s) \phi_{n} a_{n} z^{n}, \\
& D_{\lambda, \delta}^{\sigma, s} g_{k}(z)=\sum_{n=1}^{\infty} \psi_{n}(\lambda, \delta, \sigma, s) \phi_{n} b_{n} z^{n} .
\end{aligned}
$$

Further, denote by $\bar{M} H_{k}^{\sigma, s}(\lambda, \delta, \alpha)$ the subclasses of $M H_{k}^{\sigma, s}(\lambda, \delta, \alpha)$, such that the functions $h$ and $g$ in $f=h+\bar{g}$ are of the form

$$
\begin{aligned}
& h(z)=z-\sum_{n=2}^{\infty}\left|a_{n}\right| z^{n}, \\
& g(z)=\sum_{n=1}^{\infty}\left|b_{n}\right| z^{n}, \quad\left|b_{1}\right|<1,
\end{aligned}
$$

and the functions $h_{k}$ and $g_{k}$ in $f_{k}=h_{k}+\bar{g}_{k}$ are of the form

$$
\begin{aligned}
& h_{k}(z)=z-\sum_{n=2}^{\infty} \phi_{n}\left|a_{n}\right| z^{n}, \\
& g_{k}(z)=\sum_{n=1}^{\infty} \phi_{n}\left|b_{n}\right| z^{n}, \quad\left|b_{1}\right|<1 .
\end{aligned}
$$

In this paper, we obtain inclusion properties and coefficient conditions for the class $M H_{k}^{\sigma, s}(\lambda, \delta, \alpha)$. A representation theorem and distortion bounds for the class $\bar{M} H_{k}^{\sigma, s}(\lambda, \delta, \alpha)$ are also established.

Lemma 1 (see [12]). Let $f=h+\bar{g} \in S H$; if

$$
\sum_{n=2}^{\infty} \frac{1-\alpha}{2-\alpha}\left|a_{n}\right|+\sum_{n=1}^{\infty} \frac{1+\alpha}{2-\alpha}\left|b_{n}\right| \leq 1,
$$

where $0 \leq \alpha<1$, then $f$ is harmonic, sense-preserving, univalent in $U$ and $f$ is starlike harmonic of order $\alpha$.

\section{Main Results}

First, we give a meaningful conclusion about the class $M H_{k}^{\sigma, s}(\lambda, \delta, \alpha)$.

Theorem 2. Let $f \in M H_{k}^{\sigma, s}(\lambda, \delta, \alpha)$, where $f$ is given by (1); then $f_{k}$ defined by (5) is in $M H_{1}^{\sigma, s}(\lambda, \delta, \alpha)=M H^{\sigma, s}(\lambda, \delta, \alpha)$.

Proof. Let $f \in M H_{k}^{\sigma, s}(\lambda, \delta, \alpha)$. Then substituting $r e^{i \theta}$ by $\varepsilon^{v} r e^{i \theta}$, where $\varepsilon^{k}=1(\nu=0,1, \ldots, k-1)$ in (11), respectively, we have

$$
\operatorname{Im}\left\{\frac{(\partial / \partial \theta) D_{\lambda, \delta}^{\sigma, s} f\left(\varepsilon^{v} r e^{i \theta}\right)}{D_{\lambda, \delta}^{\sigma, s} f_{k}\left(\varepsilon^{\nu} r e^{i \theta}\right)}\right\} \geq \alpha .
$$

According to the definition of $f_{k}$ and $\varepsilon^{k}=1$, we know $f_{k}\left(\varepsilon^{v} r e^{i \theta}\right)=\varepsilon^{\nu} f_{k}\left(r e^{i \theta}\right)$ for any $v=0,1, \ldots, k-1$ and summing up we can get

$$
\begin{aligned}
& \operatorname{Im}\left\{\frac{1}{k} \sum_{\nu=0}^{k-1} \frac{(\partial / \partial \theta) D_{\lambda, \delta}^{\sigma, s} f\left(\varepsilon^{\nu} r e^{i \theta}\right)}{\varepsilon^{\nu} D_{\lambda, \delta}^{\sigma, s} f_{k}\left(r e^{i \theta}\right)}\right\} \\
& =\operatorname{Im}\left\{\frac{(\partial / \partial \theta) D_{\lambda, \delta}^{\sigma, s} f_{k}\left(r e^{i \theta}\right)}{D_{\lambda, \delta}^{\sigma, s} f_{k}\left(r e^{i \theta}\right)}\right\} \geq \alpha ;
\end{aligned}
$$

that is, $f_{k} \in M H^{\sigma, s}(\lambda, \delta, \alpha)$. 
Next, a sufficient coefficient condition for harmonic functions in $M H^{\sigma, s}(\lambda, \delta, \alpha)$ is given.

Theorem 3. Let $f=h+\bar{g}$ with $h$ and $g$ given by (1) and $f_{k}=h_{k}+\bar{g}_{k}$ with $h_{k}$ and $g_{k}$ given by (5). Let

$$
\begin{aligned}
\sum_{n=1}^{\infty} \psi_{(n-1) k+1}(\lambda, \delta, \sigma, s) & {\left[\frac{(n-1) k-\alpha \phi_{n}+1}{1-\alpha}\left|a_{(n-1) k+1}\right|\right.} \\
& \left.+\frac{(n-1) k+\alpha \phi_{n}+1}{1-\alpha}\left|b_{(n-1) k+1}\right|\right] \\
+ & \sum_{\substack{n=2 \\
n \neq k+1}}^{\infty} \frac{n \psi_{n}(\lambda, \delta, \sigma, s)}{1-\alpha}\left[\left|a_{n}\right|+\left|b_{n}\right|\right] \leq 2,
\end{aligned}
$$

where $a_{1}=1, \iota \geq 1$, and $\lambda \geq 0$, for $\delta, \sigma, s \in N_{0}=\{0,1,2, \ldots\}$ and $k \geq 1$. Then $f$ is sense-preserving harmonic univalent in $U$ and $f \in M H_{k}^{\sigma, s}(\lambda, \delta, \alpha)$.

Proof. Since

$$
\begin{aligned}
& \sum_{n=1}^{\infty}\left[\frac{n-\alpha}{1-\alpha}\left|a_{n}\right|+\frac{n+\alpha}{1-\alpha}\left|b_{n}\right|\right] \\
& \leq \sum_{n=1}^{\infty} \psi_{n}(\lambda, \delta, \sigma, s)\left[\frac{n-\alpha \phi_{n}}{1-\alpha}\left|a_{n}\right|+\frac{n+\alpha \phi_{n}}{1-\alpha}\left|b_{n}\right|\right] \\
& \phi_{n}=\frac{1}{k} \sum_{v=0}^{k-1} \varepsilon^{(n-1) v}, \quad \varepsilon^{k}=1, \\
& =\sum_{n=1}^{\infty} \psi_{(n-1) k+1}(\lambda, \delta, \sigma, s) \\
& \quad \times\left[\frac{(n-1) k-\alpha \phi_{n}+1}{1-\alpha}\left|a_{(n-1) k+1}\right|\right. \\
& \left.\quad+\frac{(n-1) k-\alpha \phi_{n}+1}{1-\alpha}\left|b_{(n-1) k+1}\right|\right] \\
& +\sum_{\substack{n=2 \\
n \neq t k+1}}^{\infty} \frac{n \psi_{n}(\lambda, \delta, \sigma, s)}{1-\alpha}\left[\left|a_{n}\right|+\left|b_{n}\right|\right] \leq 2,
\end{aligned}
$$

by Lemma 1 , we conclude that $f$ is sense-preserving, harmonic univalent, and starlike in $U$. To prove $f \in$ $M H_{k}^{\sigma, s}(\lambda, \delta, \alpha)$, according to the condition (11), we need to show that

$$
\begin{aligned}
\operatorname{Im}\left\{\frac{(\partial / \partial \theta) D_{\lambda, \delta}^{\sigma, s} f\left(r e^{i \theta}\right)}{D_{\lambda, \delta}^{\sigma, s} f_{k}\left(r e^{i \theta}\right)}\right\} \\
=\Re\left\{\frac{z\left(D_{\lambda, \delta}^{\sigma, s} h(z)\right)^{\prime}-(-1)^{s} z\left(D_{\lambda, \delta}^{\sigma, s} g(z)\right)^{\prime}}{D_{\lambda, \delta}^{\sigma, s} h_{k}(z)+(-1)^{s} \overline{D_{\lambda, \delta}^{\sigma, s} g_{k}(z)}}\right\} \\
=\Re \frac{A(z)}{B(z)} \geq \alpha,
\end{aligned}
$$

$$
\begin{aligned}
A(z)= & z\left(D_{\lambda, \delta}^{\sigma, s} h(z)\right)^{\prime}-(-1)^{s} z \overline{\left(D_{\lambda, \delta}^{\sigma, s} g(z)\right)^{\prime}} \\
= & z+\sum_{n=2}^{\infty} \psi_{n}(\lambda, \delta, \sigma, s) a_{n} z^{n}-(-1)^{s} \\
& \times \sum_{n=1}^{\infty} \psi_{n}(\lambda, \delta, \sigma, s) b_{n} z^{n}, \\
B(z)= & D_{\lambda, \delta}^{\sigma, s} h_{k}(z)+(-1)^{s} \overline{D_{\lambda, \delta}^{\sigma, s} g_{k}(z)} \\
= & z+\sum_{n=2}^{\infty} \psi_{n}(\lambda, \delta, \sigma, s) \phi_{n} a_{n} z^{n} \\
& +(-1)^{s} \sum_{n=1}^{\infty} \psi_{n}(\lambda, \delta, \sigma, s) \phi_{n} b_{n} z^{n},
\end{aligned}
$$

where

$$
\phi_{n}=\frac{1}{k} \sum_{\nu=0}^{k-1} \varepsilon^{(n-1) v}, \quad \varepsilon^{k}=1
$$

Using the fact that $\Re(\omega) \geq \alpha$ if and only if $|1-\alpha+\omega| \geq$ $|1+\alpha-\omega|$, it suffices to show that

$$
|A(z)+(1-\alpha) B(z)|-|A(z)-(1+\alpha) B(z)| \geq 0 .
$$

On the other hand, for $A(z)$ and $B(z)$ as given in (20) and (21), respectively, we have

$$
\begin{aligned}
\mid A & (z)+(1-\alpha) B(z)|-| A(z)-(1+\alpha) B(z) \mid \\
= & \mid(1-\alpha) D_{\lambda, \delta}^{\sigma, s} h_{k}(z)+z\left(D_{\lambda, \delta}^{\sigma, s} h(z)\right)^{\prime} \\
& +(-1)^{s}\left[\overline{(1-\alpha) D_{\lambda, \delta}^{\sigma, s} g_{k}(z)-z\left(D_{\lambda, \delta}^{\sigma, s} g(z)\right)^{\prime}}\right] \mid \\
& -\mid(1+\alpha) D_{\lambda, \delta}^{\sigma, s} h_{k}(z)-z\left(D_{\lambda, \delta}^{\sigma, s} h(z)\right)^{\prime} \\
& +(-1)^{s}\left[\overline{(1+\alpha) D_{\lambda, \delta}^{\sigma, s} g_{k}(z)+z\left(D_{\lambda, \delta}^{\sigma, s} g(z)\right)^{\prime}}\right] \mid \\
= & \mid(2-\alpha) z+\sum_{n=2}^{\infty} \psi_{n}(\lambda, \delta, \sigma, s)\left[n+(1-\alpha) \phi_{n}\right] a_{n} z^{n} \\
& -(-1)^{s} \sum_{n=2}^{\infty} \psi_{n}(\lambda, \delta, \sigma, s)\left[n-(1-\alpha) \phi_{n}\right] b_{n} z^{n} \mid \\
& -\mid \begin{array}{l}
-\alpha z+\sum_{n=2}^{\infty} \psi_{n}(\lambda, \delta, \sigma, s)\left[n-(1+\alpha) \phi_{n}\right] a_{n} z^{n} \\
\quad \\
\quad-(-1)^{s} \sum_{n=2}^{\infty} \psi_{n}(\lambda, \delta, \sigma, s)\left[n+(1+\alpha) \phi_{n}\right] b_{n} z^{n} \mid
\end{array}
\end{aligned}
$$




$$
\begin{aligned}
& \geq(2-\alpha)|z|-\sum_{n=2}^{\infty} \psi_{n}(\lambda, \delta, \sigma, s)\left[n+(1-\alpha) \phi_{n}\right]\left|a_{n}\right||z|^{n} \\
& -\sum_{n=1}^{\infty} \psi_{n}(\lambda, \delta, \sigma, s)\left[n+(1-\alpha) \phi_{n}\right]\left\|b_{n}\right\||z|^{n} \\
& -\alpha|z|-\sum_{n=2}^{\infty} \psi_{n}(\lambda, \delta, \sigma, s)\left[n-(1+\alpha) \phi_{n}\right]\left|a_{n}\right||z|^{n} \\
& -\sum_{n=1}^{\infty} \psi_{n}(\lambda, \delta, \sigma, s)\left[n+(1+\alpha) \phi_{n}\right]\left|b_{n}\right||z|^{n} \\
& =(2-\alpha)|z|\left\{1-\sum_{n=2}^{\infty} \psi_{n}(\lambda, \delta, \sigma, s)\left[\frac{n-\alpha \phi_{n}}{1-\alpha}\right]\left|a_{n}\right||z|^{n-1}\right. \\
& \left.-\sum_{n=1}^{\infty} \psi_{n}(\lambda, \delta, \sigma, s)\left[\frac{n+\alpha \phi_{n}}{1-\alpha}\right]\left|b_{n}\right||z|^{n-1}\right\} \\
& \geq 2(1-\alpha)\left\{1-\sum_{n=2}^{\infty} \psi_{n}(\lambda, \delta, \sigma, s)\left[\frac{n-\alpha \phi_{n}}{1-\alpha}\right]\left|a_{n}\right|\right. \\
& \left.-\sum_{n=1}^{\infty} \psi_{n}(\lambda, \delta, \sigma, s)\left[\frac{n+\alpha \phi_{n}}{1-\alpha}\right]\left|b_{n}\right|\right\} \geq 0
\end{aligned}
$$

Note that, by substituting the value of $\phi_{n}$ given by (7) in the previous inequality above, then

$$
\begin{aligned}
|A(z)+(1-\alpha) B(z)|-|A(z)-(1+\alpha) B(z)| \\
\geq 2(1-\alpha)\left\{1-\sum_{n=1}^{\infty} \psi_{n}(\lambda, \delta, \sigma, s)\right. \\
\times\left[\frac{(n-1) k-\alpha+1}{1-\alpha}\left|a_{(n-1) k+1}\right|\right. \\
\left.\quad-\frac{(n-1) k+\alpha+1}{1-\alpha}\left|b_{(n-1) k+1}\right|\right] \\
\left.+\sum_{\substack{n=2 \\
n \neq l k+1}}^{\infty} \frac{n \psi_{n}(\lambda, \delta, \sigma, s)}{1-\alpha}\left[\left|a_{n}\right|+\left|b_{n}\right|\right]\right\} \geq 0,
\end{aligned}
$$

by (18). Thus concludes the proof of the theorem.

Next, the condition (18) is also necessary for functions in $\bar{M} H_{k}^{\sigma, s}(\lambda, \delta, \alpha)$, which is clarified.

Theorem 4. Let $f=h+\bar{g}$ with $h$ and g given by (13) and $f_{k}=$ $h_{k}+\bar{g}_{k}$ with $h_{k}$ and $g_{k}$ given by (14). Then $f \in \bar{M} H_{k}^{\sigma, s}(\lambda, \delta, \alpha)$ if and only if

$$
\begin{aligned}
& \sum_{n=1}^{\infty} \psi_{(n-1) k+1}(\lambda, \delta, \rho, s) \\
& \quad \times\left[\frac{(n-1) k-\alpha \phi_{n}+1}{1-\alpha}\left|a_{(n-1) k+1}\right|\right.
\end{aligned}
$$

$$
\begin{gathered}
\left.+\frac{(n-1) k+\alpha \phi_{n}+1}{1-\alpha}\left|b_{(n-1) k+1}\right|\right] \\
+\sum_{\substack{n=2 \\
n \neq i k+1}}^{\infty} \frac{n \psi_{n}(\lambda, \delta, \sigma, s)}{1-\alpha}\left[\left|a_{n}\right|+\left|b_{n}\right|\right] \leq 2,
\end{gathered}
$$

where $a_{1}=1, \iota \geq 1$, and $\lambda \geq 0$, for $\delta, \sigma, s \in N_{0}=\{0,1,2, \ldots\}$ and $k \geq 1$ given by (6).

Proof. The if part follows from Theorem 3 upon noting that if the analytic and coanalytic parts of $f=h+\bar{g} \in M H_{k}^{\sigma, s}(\lambda, \delta, \alpha)$ are of the form (13), then $f \in \bar{M} H_{k}^{\sigma, s}(\lambda, \delta, \alpha)$. For the only if part, we show that $f \notin \bar{M} H_{k}^{\sigma, s}(\lambda, \delta, \alpha)$, if the condition (26) does not hold. Thus we can write

$$
\mathfrak{R}\left\{\frac{z\left(D_{\lambda, \delta}^{\sigma, s} h(z)\right)^{\prime}-(-1)^{s} z\left(D_{\lambda, \delta}^{\sigma, s} g(z)\right)^{\prime}}{D_{\lambda, \delta}^{\sigma, s} h_{k}(z)+(-1)^{s} \overline{D_{\lambda, \delta}^{\sigma, s} g_{k}(z)}}\right\} \geq \alpha
$$

this is equivalent to

$$
0 \leq \Re\left\{\frac{z\left(D_{\lambda, \delta}^{\sigma, s} h(z)\right)^{\prime}-(-1)^{s} z \overline{\left(D_{\lambda, \delta}^{\sigma, s} g(z)\right)^{\prime}}}{D_{\lambda, \delta}^{\sigma, s} h_{k}(z)+(-1)^{s} \overline{D_{\lambda, \delta}^{\sigma, s} g_{k}(z)}}\right\}-\alpha .
$$

That is,

$$
\begin{gathered}
\Re\left\{\left(\begin{array}{l}
(1-\alpha) z-\sum_{n=2}^{\infty} \psi_{n}(\lambda, \delta, \sigma, s)\left(n-\alpha \phi_{n}\right)\left|a_{n}\right| z^{n} \\
\quad-(-1)^{s} \sum_{n=1}^{\infty} \psi_{n}(\lambda, \delta, \sigma, s)\left(n+\alpha \phi_{n}\right)\left|b_{n}\right| z^{n}
\end{array}\right.\right. \\
\quad \times\left(z-\sum_{n=2}^{\infty} \psi_{n}(\lambda, \delta, \sigma, s) \phi_{n}\left|a_{n}\right| z^{n}\right. \\
\left.\left.\quad+(-1)^{s} \overline{\sum_{n=1}^{\infty} \psi_{n}(\lambda, \delta, \sigma, s) \phi_{n}\left|b_{n}\right| z^{n}}\right)^{-1}\right\} \\
\geq 0 .
\end{gathered}
$$

The above-required condition must hold for all values of $z$, $|z|=r<1$. Upon choosing the values of $z$ on the positive real axis where $0 \leq z=r<1$, we must have

$$
\begin{gathered}
\left((1-\alpha)-\sum_{n=2}^{\infty} \psi_{n}(\lambda, \delta, \sigma, s)\left(n-\alpha \phi_{n}\right)\left|a_{n}\right| r^{n-1}\right. \\
\left.-\sum_{n=1}^{\infty} \psi_{n}(\lambda, \delta, \sigma, s)\left(n+\alpha \phi_{n}\right)\left|b_{n}\right| r^{n-1}\right)
\end{gathered}
$$




$$
\begin{gathered}
\times\left(1-\sum_{n=2}^{\infty} \psi_{n}(\lambda, \delta, \sigma, s) \phi_{n}\left|a_{n}\right| r^{n-1}\right. \\
\left.+\sum_{n=1}^{\infty} \psi_{n}(\lambda, \delta, \sigma, s) \phi_{n}\left|b_{n}\right| r^{n-1}\right)^{-1}
\end{gathered}
$$$$
\geq 0 \text {. }
$$

If the condition (26) does not hold, then the numerator in (30) is negative for $r$ sufficiently close to 1 . Hence there exists a $z_{0}=r_{0}$ in $(0,1)$ for which the quotient in (30) is negative. This contradicts the required condition for $f \in \bar{M} H_{k}^{\sigma, s}(\lambda, \delta, \alpha)$ and the proof is complete.

Now, the distortion result is given.

Theorem 5. If $f \in \bar{M} H_{k}^{\sigma, s}(\lambda, \delta, \alpha)$, then

$$
\begin{aligned}
& |f(z)| \geq\left(1-\left|b_{1}\right|\right)-\frac{1}{\psi_{2}(\lambda, \delta, \sigma, s)}\left[\frac{1-\alpha}{2-\alpha}-\frac{1+\alpha}{2-\alpha}\left|b_{1}\right|\right] r^{2}, \\
& |f(z)| \leq\left(1+\left|b_{1}\right|\right)+\frac{1}{\psi_{2}(\lambda, \delta, \sigma, s)}\left[\frac{1-\alpha}{2-\alpha}-\frac{1+\alpha}{2-\alpha}\left|b_{1}\right|\right] r^{2} .
\end{aligned}
$$

Proof. We will only prove the left-hand inequality of the above theorem. The arguments for the right-hand inequality are similar and so we omit it. Let $f \in \bar{M} H_{k}^{\sigma, s}(\lambda, \delta, \alpha)$. Taking the absolute value of $f(z)$ we obtain

$$
\begin{aligned}
|f(z)| \geq & \left(1-\left|b_{1}\right|\right) r-\sum_{n=2}^{\infty}\left[\left|a_{n}\right|+\left|b_{n}\right|\right] r^{n} \\
\geq & \left(1-\left|b_{1}\right|\right) r-\sum_{n=2}^{\infty}\left[\left|a_{n}\right|+\left|b_{n}\right|\right] r^{2} \\
\geq & \left(1-\left|b_{1}\right|\right) r-\frac{1-\alpha}{\psi_{2}(\lambda, \delta, \rho, s)\left(2-\alpha \phi_{2}\right)} \\
& \times \sum_{n=2}^{\infty} \frac{\psi_{2}(\lambda, \delta, \rho, s)\left(2-\alpha \phi_{2}\right)}{1-\alpha}\left[\left|a_{n}\right|+\left|b_{n}\right|\right] r^{2} \\
\geq & \left(1-\left|b_{1}\right|\right) r-\frac{1-\alpha}{2-\alpha} \sum_{n=2}^{\infty} \psi_{n}(\lambda, \delta, \rho, s) \\
& \times\left[\frac{\left(n-\alpha \phi_{n}\right)}{1-\alpha}\left|a_{n}\right|+\frac{\left(n+\alpha \phi_{n}\right)}{1-\alpha}\left|b_{n}\right|\right] r^{2}
\end{aligned}
$$

by (26):

$$
=\left(1-\left|b_{1}\right|\right)-\frac{1}{\psi_{2}(\lambda, \delta, \sigma, s)}\left[\frac{1-\alpha}{2-\alpha}-\frac{1+\alpha}{2-\alpha}\left|b_{1}\right|\right] r^{2} .
$$

The following covering result follows from the left-hand inequality in Theorem 5.
Corollary 6. If $f \in \bar{M} H_{k}^{\sigma, s}(\lambda, \delta, \alpha)$ then

$$
\begin{aligned}
\{\omega:|\omega|< & \frac{2 \psi_{2}-1-\left(\psi_{2}(\lambda, \delta, \rho, s)-1\right) \alpha}{\psi_{2}(\lambda, \delta, \rho, s)(2-\alpha)} \\
& \left.-\frac{2 \psi_{2}(\lambda, \delta, \rho, s)-1-\left(\psi_{2}(\lambda, \delta, \rho, s)+1\right) \alpha}{\psi_{2}(\lambda, \delta, \rho, s)(2-\alpha)}\left|b_{1}\right|\right\}
\end{aligned}
$$

$\subset f(U)$.

Note that other work related to Sakaguchi and classes of functions with respect to symmetric points can be found in [13-16].

\section{Conflict of Interests}

The authors declare that they have no competing interests.

\section{Authors' Contribution}

Both authors read and approved the final paper.

\section{Acknowledgments}

The work presented here was partially supported by AP-2013009 and DIP-2013-001. The authors also would like to thank the referees for the comments given to improve the paper.

\section{References}

[1] J. Clunie and T. Sheil-Small, "Harmonic univalent functions," Annales Academiae Scientiarum Fennicae A I: Mathematica, vol. 9, pp. 3-25, 1984.

[2] T. Sheil-Small, "Constants for planar harmonic mappings," Journal of the London Mathematical Society, vol. 42, no. 2, pp. 237-248, 1990.

[3] K. Sakaguchi, "On a certain univalent mapping," Journal of the Mathematical Society of Japan, vol. 11, pp. 72-75, 1959.

[4] T. N. Shanmugam, C. Ramachandran, and V. Ravichandran, "Fekete-Szegö problem for subclasses of starlike functions with respect to symmetric points," Bulletin of the Korean Mathematical Society, vol. 43, no. 3, pp. 589-598, 2006.

[5] R. Chand and P. Singh, "On certain schlicht mappings," Indian Journal of Pure and Applied Mathematics, vol. 10, no. 9, pp. 1167$1174,1979$.

[6] R. N. das and P. Singh, "On subclasses of schlicht mapping," Indian Journal of Pure and Applied Mathematics, vol. 8, no. 8, pp. 864-872, 1977.

[7] O. P. Ahuja and J. M. Jahangiri, "Sakaguchi-type harmonic univalent functions," Scientiae Mathematicae Japonicae, vol. 59, no. 1, pp. 239-244, 2004.

[8] K. Al Shaqsi and M. Darus, "On subclass of harmonic starlike functions with respect to $K$-symmetric points," International Mathematical Forum, vol. 2, no. 57-60, pp. 2799-2805, 2007.

[9] A. A. Ali Abubaker and M. Darus, "On starlike and convex functions with respect to $k$-symmetric points," International Journal of Mathematics and Mathematical Sciences, vol. 2011, Article ID 834064, 9 pages, 2011. 
[10] J. M. Jahangiri, G. Murugusundaramoorthy, and K. Vijaya, "Salagean-type harmonic univalent functions," Southwest Journal of Pure and Applied Mathematics, no. 2, pp. 77-82, 2002.

[11] K. Al-Shaqsi and M. Darus, "On univalent functions with respect to $k$-symmetric points defined by a generalized Ruscheweyh derivatives operator," Journal of Analysis and Applications, vol. 7, no. 1, pp. 53-61, 2009.

[12] J. M. Jahangiri, "Harmonic functions starlike in the unit disk," Journal of Mathematical Analysis and Applications, vol. 235, no. 2, pp. 470-477, 1999.

[13] A. Abubaker and M. Darus, "A note on subclass of analytic functions with respect to $k$-symmetric points," International Journal of Mathematical Analysis, vol. 6, no. 12, pp. 573-589, 2012.

[14] K. Al-Shaqsi and M. Darus, "Application of Hölder inequality in generalised convolutions for functions with respect to $k$ symmetric points," Applied Mathematical Sciences, vol. 3, no. 3336, pp. 1787-1797, 2009.

[15] M. Darus and R. W. Ibrahim, "Analytic functions with respect to $\mathrm{N}$-symmetric, conjugate and symmetric conjugate points involving generalized differential operator," Proceedings of the Pakistan Academy of Sciences, vol. 46, no. 2, pp. 75-83, 2009.

[16] B. A. Frasin and M. Darus, "Subordination results on subclasses concerning Sakaguchi functions," Journal of Inequalities and Applications, vol. 2009, Article ID 574014, 7 pages, 2009. 


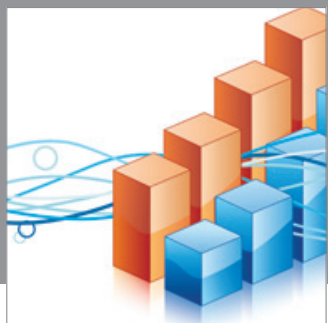

Advances in

Operations Research

mansans

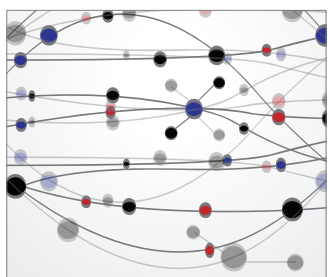

The Scientific World Journal
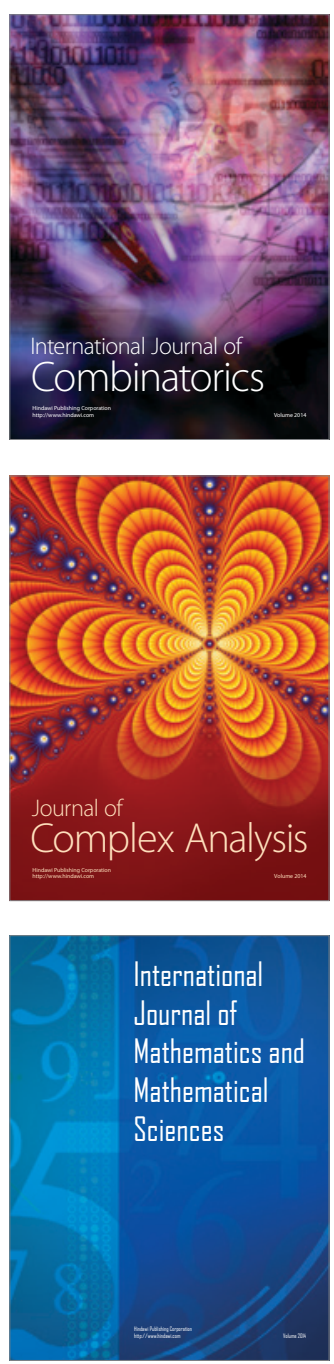
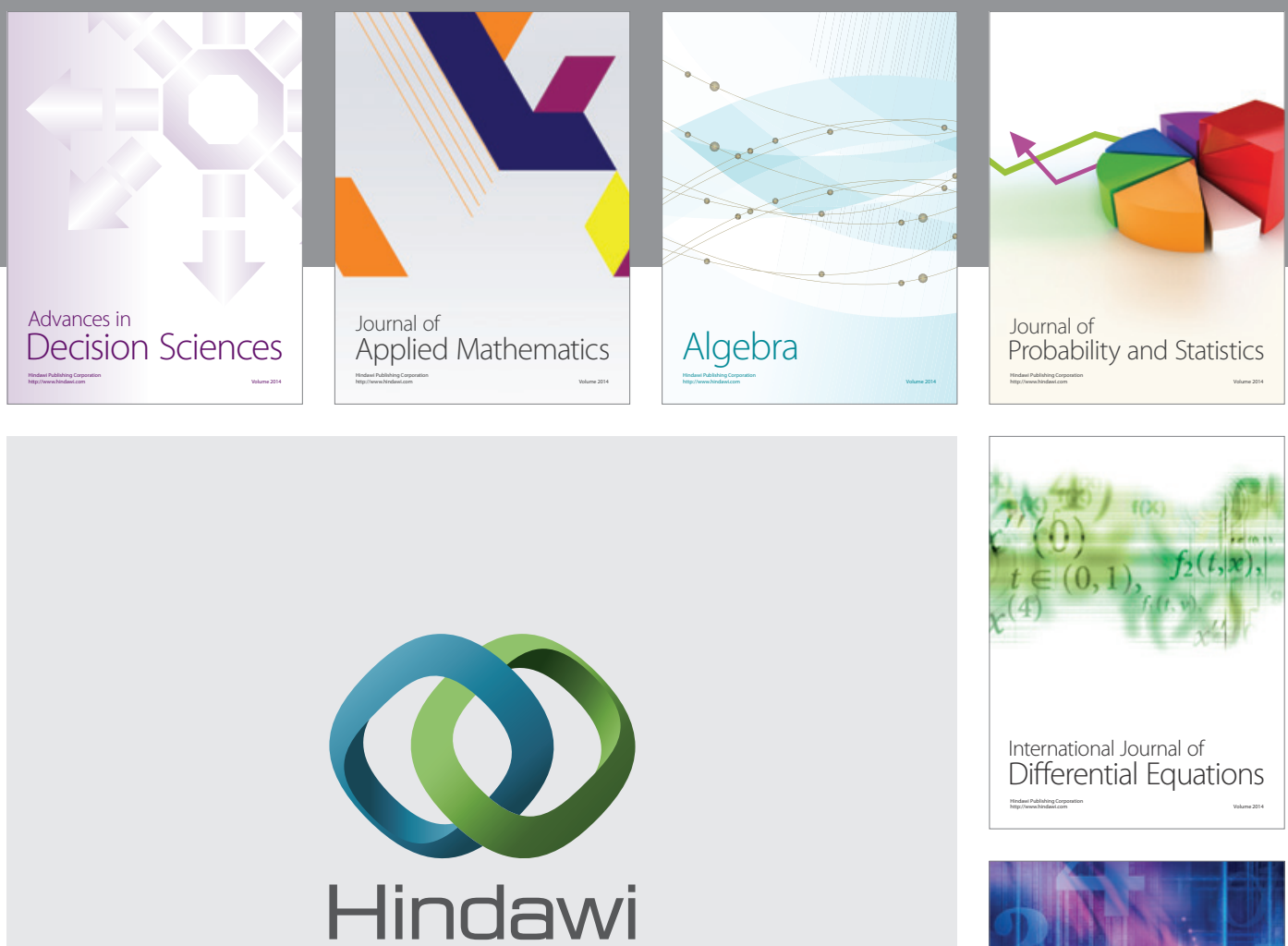

Submit your manuscripts at http://www.hindawi.com
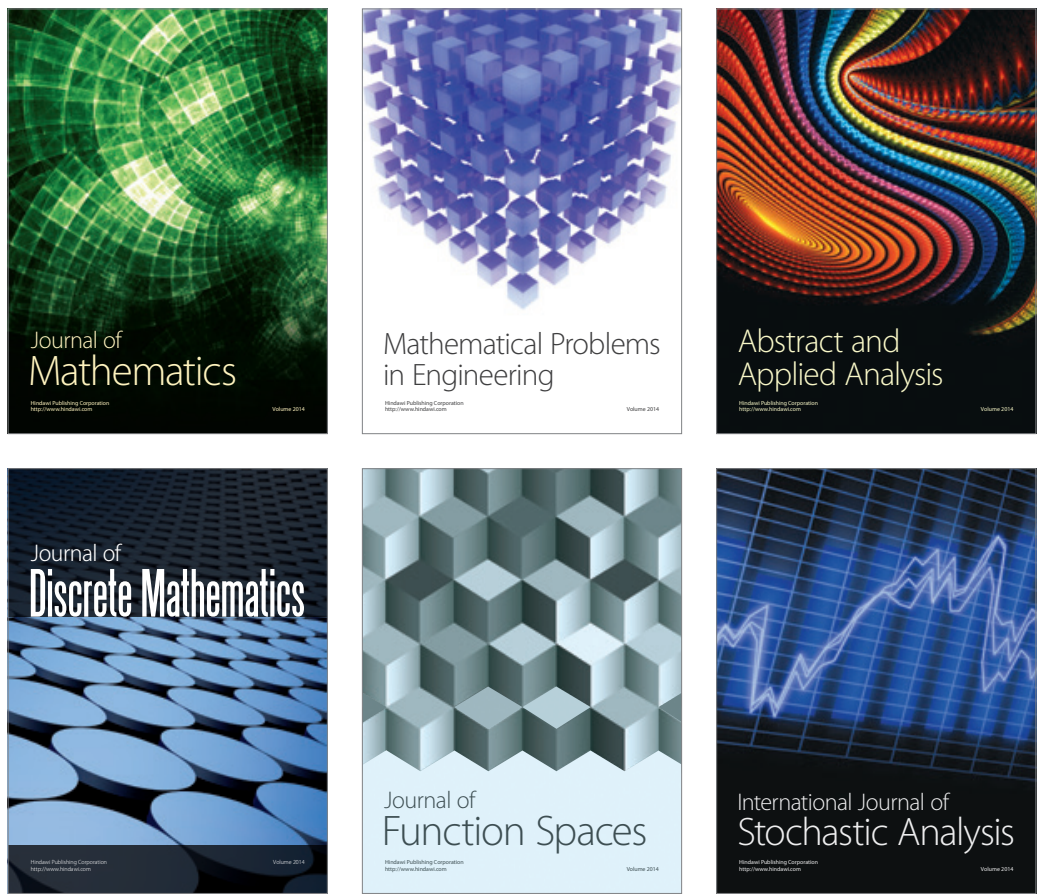

Journal of

Function Spaces

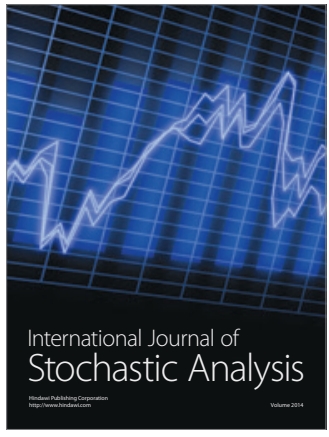

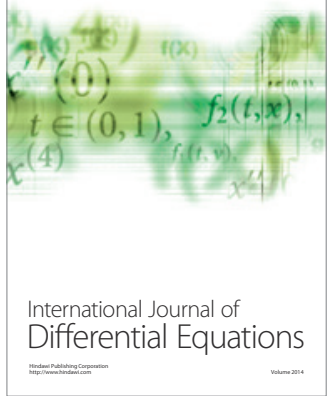
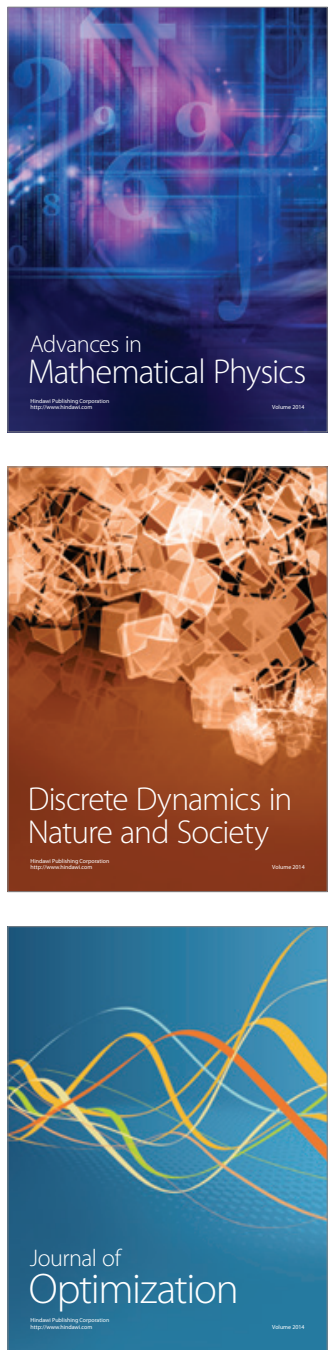